PROCEEDINGS OF THE

AMERICAN MATHEMATICAL SOCIETY

Volume 126, Number 5, May 1998, Pages 1363-1370

S $0002-9939(98) 04068-4$

\title{
ON ANALYTIC STRUCTURE OF SOLUTIONS TO HIGHER ORDER ABSTRACT CAUCHY PROBLEMS
}

\author{
YULIA MISHURA AND YURI TOMILOV
}

(Communicated by Palle E. T. Jorgensen)

\begin{abstract}
We prove the existence of entire solutions to some abstract higher order Cauchy problem for a dense subset of initial values.
\end{abstract}

Let $X$ be a complex Banach space, and let $A$ be a closed linear operator on $X$ with dense domain $D(A)$. We study analytic properties of strong solutions of the higher order Cauchy problem

$$
\begin{gathered}
x^{(p)}(t)=A x(t), t \in[0, \infty), \\
x^{(i)}(0)=x_{i}, 0 \leq i \leq p-1, p \in \mathbb{N} .
\end{gathered}
$$

These questions were considered for the cases $p=1,2$ and for uniformly well-posed Cauchy problems in the papers $[3,6,7]$ (and related topics in [1]). The special properties of semigroups and cosine (sine) functions that correspond to such Cauchy problems were used in the arguments. But in the case $p \geq 3$ the Cauchy problem (1) is uniformly well-posed if and only if $A$ is a bounded operator [3].

So it is natural to treat the problem (1) in its intrinsic terms and essentially weaken well-posedness assumptions.

Let us consider a particular case of the problem (1):

$$
\begin{gathered}
x^{(p)}(t)=A x(t), t \in[0, \infty), \\
x^{(i)}(0)=0,1 \leq i \leq p-1, x(0)=x_{0} .
\end{gathered}
$$

Definition 1. We say that a closed densely defined linear operator $A$ satisfies the condition $(G)$ if the set $E=\left\{x_{0} \in D(A) \mid\right.$ there exists a solution $x\left(t, x_{0}\right)$ of the problem (2) such that

$$
\left\|x^{(p)}\left(t, x_{0}\right)\right\| \leq C e^{\alpha t^{p}}, \quad t \geq 0
$$

for some $\alpha=\alpha\left(x_{0}\right)>0$ and some $\left.C>0\right\}$ is dense in $X$.

Remark 1. If $p=2$ and $A$ is a generator of the strongly continuous cosine function, we can put $E=D(A)$.

Remark 2 . The inequality (3) implies that the solution $x\left(t, x_{0}\right)$ of $(2)$ also satisfies the inequalities

$$
\max _{0 \leq k \leq p}\left\|x^{(k)}\left(t, x_{0}\right)\right\| \leq C_{1} e^{\beta t^{p}} \text { for some } \beta=\beta\left(x_{0}\right)>0 .
$$

Received by the editors November 13, 1995 and, in revised form, October 14, 1996.

1991 Mathematics Subject Classification. Primary 34G10, 47D09, 47A50.

Key words and phrases. Abstract Cauchy problem, entire solution.

(C) 1998 American Mathematical Society 
Indeed, (4) follows from the identity

$$
x^{(k)}(t)=\frac{1}{(p-k-1) !} \int_{0}^{t}(t-s)^{p-k-1} x^{(p)}(s) d s, \quad 0 \leq k \leq p-1 .
$$

The following two examples illustrate the condition $(G)$.

Example 1. We shall show that the condition $(G)$ is not too restrictive. Let $A$ be a closed densely defined operator satisfying the assumption:

there exist $C_{0}>0,0<a<1$ and $b_{0}$ such that $R(z, A)$, the resolvent of

$\left(G_{1}\right) A$, exists for $\operatorname{Re} z \geq \max \left\{b_{0}, c_{0}|\operatorname{Im} z|^{a}\right\}$, and $\|R(z, A)\| \leq M\left(1+|z|^{N}\right)$, $M>0, N \geq 0$, for these $z$.

Define

$$
\begin{aligned}
& \|x\|_{\beta, \varepsilon}=\sup _{n \in \mathbb{N}}(n !)^{-\beta} \varepsilon^{n}\left\|A^{n} x\right\|, \quad x \in C^{\infty}(A)=\bigcap_{n=1}^{\infty} D\left(A^{n}\right), \\
& G(\beta, \varepsilon)=\left\{x \in X:\|x\|_{\beta, \varepsilon}<\infty\right\}, \quad G(\beta)=\bigcup_{\varepsilon>0} G(\beta, \varepsilon) .
\end{aligned}
$$

Then, according to [1], $\overline{G(\beta)}=X, \beta>1$. So the set $M=\left\{x \in C^{\infty}(A)\|\| A^{n} x \| \leq\right.$ $\alpha^{n-1} n^{n(p-1)}, n \geq 1$, for some $\left.\alpha=\alpha(x)>0\right\}$ is dense in $X$. Moreover, for $x \in M$ we have

$$
\begin{aligned}
\sum_{n=0}^{\infty} \frac{t^{n p}}{(n p) !}\left\|A^{n+1} x\right\| & \leq \sum_{n=0}^{\infty} \frac{t^{n p}}{(n p) !} \alpha^{n}(n+1)^{(n+1)(p-1)} \\
& \leq C \sum_{n=0}^{\infty} \frac{\left(\alpha_{1} t^{p}\right)^{n}}{n !}=C e^{\alpha_{1} t^{p}}, \quad \alpha_{1}=\alpha_{1}(x)>0 .
\end{aligned}
$$

It is easy to see that the function

$$
x\left(t, x_{0}\right)=\sum_{n=0}^{\infty} \frac{t^{n p}}{(n p) !} A^{n} x_{0}, x_{0} \in M,
$$

is a solution of the Cauchy problem (2) and

$$
\left\|x^{(p)}\left(t, x_{0}\right)\right\|=\left\|A x\left(t, x_{0}\right)\right\| \leq C e^{\alpha t^{p}} .
$$

Therefore, the condition $\left(G_{1}\right)$ is sufficient for $(G)$. (But it is not necessary. See example 2(b). )

Example 2(a). Now we shall demonstrate that the condition $(G)$ indeed limits the growth of solutions of (1). To this aim we modify an idea of the example from [5] to higher order equations. Set

$$
\begin{array}{r}
X_{0}:=1 . \text { s. }\left\{s^{i} e^{\sum_{j=0}^{p} a_{j} s^{j}} \mid a_{p}>0, \quad i \in \mathbb{N} \cup\{0\},\right. \\
\left.a_{j} \in \mathbb{C}, \quad 0 \leq j \leq p, \quad s \in[0,1]\right\}
\end{array}
$$

and note that $\bar{X}_{0}=C([0,1])$ in the max-norm.

Define the operator $A$ in $C([0,1])$ as the closure of $\frac{d^{p}}{d s^{p}}$ on $X_{0} . A$ is a closed linear operator with dense domain. Next consider the Cauchy problem $(2)$ in $C([0,1])$. 
Then the function $x_{0}(s)$ from $X$ belongs to $D(A)$ and $y:[0, \infty) \rightarrow C([0,1])$, given by

$$
y(t):=\frac{1}{p} \sum_{k=0}^{p-1} x_{0}\left(\omega^{k} t+s\right), \quad t \geq 0, \quad s \in[0,1],
$$

$\omega$ being a $p$-th root of unity, is a strong solution of the problem (2). Indeed,

$$
\begin{aligned}
y^{(p)}(t) & =\frac{1}{p} \sum_{k=0}^{p-1} \omega^{k p} x_{0}^{(p)}\left(\omega^{k} t+s\right)=\frac{1}{p} \sum_{k=0}^{p-1} x_{0}^{(p)}\left(\omega^{k} t+s\right) \\
& =\frac{1}{p} \sum_{k=0}^{p-1} \frac{d^{p}}{d s^{p}} x_{0}\left(\omega^{k} t+s\right)=A y(t), \quad t \geq 0, \\
\frac{d^{i}}{d t^{i}} y(0+) & =\frac{1}{p} \sum_{k=0}^{p-1} \omega^{k i} x_{0}(s)=0, \quad 1 \leq i \leq p-1, y(0)=x_{0}(s) .
\end{aligned}
$$

Moreover, by routine estimations one can prove that

$$
\left\|y^{(i)}(t)\right\| \geq C_{i} e^{\alpha t^{p}}
$$

$C_{i}>0,0 \leq i \leq p, \alpha>0$, for sufficiently large $t$. In a similar way we can indicate a Cauchy problem of the form (2) for which some solutions grow faster then $e^{t^{p}}$ as $t \rightarrow \infty$ (take, for instance,

$$
\begin{array}{r}
X=1 . \text { s. }\left\{s^{i} e^{\sum_{j=0}^{2 p} a_{j} s^{j}} \mid a_{2 p}>0, \quad i \in \mathbb{N} \cup\{0\},\right. \\
\left.a_{j} \in \mathbb{C}, \quad 0 \leq j \leq 2 p, \quad s \in[0,1]\right\}
\end{array}
$$

and the same operator $A$ in $C([0,1]))$.

Example 2(b). Next we give an example showing that the implication $(G) \Rightarrow$ $\left(G_{1}\right)$ is false. The construction is close to the preceding one. We describe it briefly. Let $X=C([0,1]), A$ the closure of $\frac{d^{p}}{d s^{p}}$, defined on the functions $\{f \in$ $\left.C([0,1]) \mid f^{(p)} \in C([0,1])\right\}$. The operator $A$ satisfies condition $(G)$ with the set $E=\left\{\sum_{n=0}^{m} a_{n} s^{n}, s \in[0,1], m \in \mathbb{N}\right.$ is arbitrary $\}$. Solutions of problem (2) can be derived by means of formula (5). On the other hand, the spectrum of $A$ fills the whole complex plane. So the condition $\left(G_{1}\right)$ is not satisfied.

Remark 3. Thus introduction of the condition $(G)$ allows us to treat the Cauchy problem (2), for which the resolvent set of the operator $A$ is empty.

The next theorem partially generalizes the main statements of $[1,2,7]$ and is proved following the general idea of [2].

Theorem 1. Let the operator A satisfy the condition $(G), p \geq 2$. Then the set

$$
\begin{aligned}
& \left\{x_{0} \in X \mid \text { the solution } x\left(t, x_{0}\right) \text { of }(2)\right. \text { exists and } \\
& \quad \text { can be extended to an entire function } x(z): \mathbb{C} \rightarrow X\}
\end{aligned}
$$

is dense in $X$.

The proof of the theorem depends on the following result. 
Lemma 1. Let $f: \mathbb{R}^{+} \rightarrow X$ be a strongly measurable function satisfying the condition

$$
\|f(t)\| \leq C e^{a t^{p}}, \quad t \geq 0, \quad a>0, \quad C>0 .
$$

Then for every $b>a$

$$
\left\|\int_{0}^{\infty}\left(e^{-b t^{p}}\right)^{(p n)} f(t) d t\right\|^{1 / n}=O\left(n^{p-1}\right), \quad n \rightarrow \infty .
$$

Proof. Observe that the next representation is true for every $m \in \mathbb{N}$ and $b=1$ :

$$
\left(e^{-t^{p}}\right)^{(m)}=e^{-t^{p}} \sum_{i=r_{m}}^{m} a_{i, m} t^{i p-m},
$$

where $r_{m}=\frac{m}{p}$ if $\frac{m}{p} \in \mathbb{N}$ and $r_{m}=\left[\frac{m}{p}\right]+1$ otherwise. Then from (6)

$$
\left(e^{-t^{p}}\right)^{(m+1)}=e^{-t^{p}} \sum_{i=r_{m+1}}^{m} a_{i, m+1} t^{i p-m-1},
$$

and, on the other hand,

$$
\begin{aligned}
\left(e^{-t^{p}}\right)^{(m+1)} & =\sum_{i=r_{m+1}}^{m}\left(-p a_{i-1, m}+a_{i, m}(i p-m)\right) t^{i p-m-1} \\
& +a_{r_{m}, m}\left(t^{r_{m} p-m}\right)^{\prime}-a_{m m} t^{(m+1) p-m-1} .
\end{aligned}
$$

Let's constitute the recurrent relations for $a_{m-k, m}, 0 \leq k \leq m-r_{m}$. We have

$$
a_{m-k, m+1}=-p a_{m-k-1, m}+a_{m-k, m}[(m-k) p-m], \quad k<m-r_{m} .
$$

If $\frac{m}{p} \notin \mathbb{N}$, then $r_{m+1}=r_{m}$ and

$$
a_{r_{m+1}, m+1}=a_{r_{m}, m}\left(r_{m} p-m\right) .
$$

(Note that $\left|r_{m} p-m\right| \leq p$.) In the opposite case $r_{m+1}=r_{m}+1$ and

$$
a_{r_{m}, m+1}=p\left(a_{r_{m+1}, m}-a_{r_{m}, m}\right) .
$$

Further, we shall prove the following inequalities:

$$
\begin{gathered}
\left|a_{m-k, m}\right| \leq \frac{p^{m} m^{2 k}}{(2 k) ! !}, \quad m \geq 1,0 \leq k<m-r_{m}, \\
\left|a_{r_{m}, m}\right| \leq \frac{p^{m}(m-1)^{2\left(m-r_{m}-1\right)}}{\left(2\left(m-r_{m}-1\right)\right) ! !}, \quad m \geq 2,
\end{gathered}
$$

where $(2 n) ! !:=2 \cdot 4 \cdot 6 \cdot \ldots \cdot 2 n, n \in \mathbb{N}$. Proceed by induction on $m$. The inequalities (12), (13) are obviously true for $m=1,2$. Suppose that they hold for every $m \leq m_{0}$ and every $0 \leq k \leq m-r_{m}$. For the next reasoning one has to consider two cases. 
1. If $a_{m_{0}+1-k, m_{0}+1}$ has the form $a_{r_{m_{0}+1}, m_{0}+1}\left(k=m_{0}+1-r_{m_{0}+1}\right)$, we use the relations (10), (11) for the estimates. For $\frac{m_{0}}{p} \notin \mathbb{N}$ we get from (13)

$$
\begin{aligned}
\left|a_{r_{m_{0}+1}, m_{0}+1}\right| & \leq\left|a_{r_{m_{0}}, m_{0}}\right| p \leq p^{m_{0}+1} \frac{\left(m_{0}-1\right)^{2\left(m_{0}-r_{m_{0}}-1\right)+1}}{\left[2\left(m_{0}-r_{m_{0}}-1\right)\right] ! !} \\
& \leq p^{m_{0}+1} \frac{m_{0}^{2\left(\left(m_{0}+1\right)-r_{m_{0}+1}-1\right)}}{\left[2\left(\left(m_{0}+1\right)-r_{m_{0}+1}-1\right)\right] ! !} .
\end{aligned}
$$

If $\frac{m_{0}}{p} \in \mathbb{N}$, then similarly from (12), (13)

$$
\begin{gathered}
\left|a_{r_{m_{0}+1}, m_{0}+1}\right| \leq p\left(\left|a_{r_{m_{0}+1}, m_{0}}\right|+\left|a_{r_{m_{0}}, m_{0}}\right|\right) \\
\leq p\left(p^{m_{0}} \frac{m_{0}^{2\left(m_{0}-r_{m_{0}}-1\right)}}{\left[2\left(m_{0}-r_{m_{0}}-1\right)\right] ! !}+p^{m_{0}}\left(m_{0}-1\right) \frac{\left(m_{0}-1\right)^{2\left(m_{0}-r_{m_{0}}-1\right)}}{\left[2\left(m_{0}-r_{m_{0}}-1\right)\right] ! !}\right) \\
\leq p^{m_{0}+1} m_{0} \frac{m_{0}^{2\left(\left(m_{0}+1\right)-r_{m_{0}+1}-1\right)}}{\left[2\left(\left(m_{0}+1\right)-r_{m_{0}+1}-1\right)\right] ! !}
\end{gathered}
$$

2. Now suppose $k<m_{0}+1-r_{m_{0}+1}$. If $k=0$, then $a_{m_{0}+1, m_{0}+1}=(-p)^{m_{0}+1}$. Thus (12) is satisfied. For fixed $m_{0}$ we use induction again. Assume that (12) holds for some $k_{0}$ and pass from $k_{0}$ to $k_{0}+1$. From (9)

$$
\begin{gathered}
a_{\left(m_{0}+1\right)-\left(k_{0}+1\right), m_{0}+1}=a_{m_{0}-k_{0}, m_{0}+1}=-p a_{m_{0}-k_{0}-1, m_{0}} \\
\left.+a_{m_{0}-k_{0}, m_{0}}\left(\left(m_{0}-k_{0}\right) p-m_{0}\right)=a_{m_{0}-k_{0}, m_{0}}\left(\left(m_{0}-k_{0}\right) p-m_{0}\right)\right) \\
-p a_{m_{0}-k_{0}-1, m_{0}-1} \times\left(\left(m_{0}-k_{0}-1\right) p-m_{0}+1\right) \\
+p^{2} a_{m_{0}-k_{0}-1, m_{0}-1}=\cdots \\
=\sum_{i=0}^{m_{0}-s}(-p)^{i} a_{m_{0}-k_{0}-i, m_{0}-i}\left(\left(m_{0}-k_{0}-i\right) p-m_{0}+i\right) \\
+(-p)^{m_{0}-s+1} a_{r_{s}, s}, \quad s-r_{s}=k_{0}+1 .
\end{gathered}
$$

By the induction hypothesis $a_{m_{0}-k_{0}-i, m_{0}-i}, \quad 0 \leq i \leq m_{0}-s$, and $a_{r_{s}, s}$ can be estimated according to (12), (13). Note also that $\left|\left(m_{0}-k_{0}-i\right) p-m_{0}+i\right| \leq\left(m_{0}-i\right) p$. So from (14), (12) and (13) we get

$$
\begin{gathered}
\left|a_{\left(m_{0}+1\right)-\left(k_{0}+1\right), m_{0}+1}\right| \leq \sum_{i=0}^{m_{0}-s} p^{i} \frac{\left(m_{0}-i\right)^{2 k_{0}}}{\left(2 k_{0}\right) ! !} p^{m_{0}-i}\left(m_{0}-i\right) p \\
+p^{m_{0}-s+1} p^{s} \frac{(s-1)^{2 k_{0}+1}}{\left(2 k_{0}\right) ! !}=p^{m_{0}+1} \sum_{i=0}^{m_{0}-s+1} \frac{\left(m_{0}-i\right)^{2 k_{0}+1}}{\left(2 k_{0}\right) ! !} \\
\leq p^{m_{0}+1} \sum_{i=0}^{m_{0}} \frac{j^{2 k_{0}+1}}{\left(2 k_{0}\right) ! !} \leq p^{m_{0}+1} \frac{\left(m_{0}+1\right)^{2\left(k_{0}+1\right)}}{\left[2\left(k_{0}+1\right)\right] ! !}
\end{gathered}
$$

Thus the required estimates (12), (13) are proved. (Observe that we shall not succeed if we substitute (12) directly in (14).) 
Further, let $m=n p$. Then $r_{m}=r_{n p}=n$ and

$$
\begin{gathered}
\left|\left(e^{-b t^{p}}\right)^{(n p)}\right| \leq e^{-b t^{p}} \max \left(1, b^{n p}\right) \sum_{i=n}^{n p}\left|a_{i, n p}\right| t^{i p-n p} \\
\leq e^{-b t^{p}} \max \left(1, b^{n p}\right) n(p-1) \max _{n \leq i \leq n p}\left|a_{i, n p}\right| t^{i p-n p}, t>0,
\end{gathered}
$$

where $a_{i, n p}, n \leq i \leq n p$, are defined by (6). So using (12), (13) we obtain:

$$
\begin{aligned}
& \left|\left(e^{-b t^{p}}\right)^{(n p)}\right| \leq e^{-b t^{p}} \max \left(1, b^{n p}\right) n(p-1) \\
& \times \max _{0 \leq k \leq n(p-1)} \frac{(n p)^{2 k} p^{n p}}{2^{k} k !} t^{(n p-k) p-n p} .
\end{aligned}
$$

By the last inequality

$$
\begin{aligned}
T & :=\left\|\int_{0}^{\infty}\left(e^{-b t^{p}}\right)^{(n p)} f(t) d t\right\| \\
& \leq C \int_{0}^{\infty}\left|\left(e^{-b t^{p}}\right)^{(n p)}\right| e^{a t^{p}} d t \leq C \max \left(1, b^{n p}\right) n(p-1) \\
& \times \max _{0 \leq k \leq n(p-1)}\left\{\frac{(n p)^{2 k} p^{n p}}{2^{k} k !} \int_{0}^{\infty} e^{(a-b) t^{p}} t^{(n p-k) p-n p} d t\right\} \\
& =C \max \left(1, b^{n p}\right) n(p-1) \max _{0 \leq k \leq n(p-1)} \frac{(n p)^{2 k}}{2^{k} k !} p^{n p-1} \\
& \times \frac{1}{(b-a)^{n p-k-n+1 / p}} \int_{0}^{\infty} e^{-z} z^{n p-k-n-1+1 / p} d z .
\end{aligned}
$$

Then (15) implies

$$
\begin{aligned}
T^{\frac{1}{n}} & \leq C_{1} \max _{0 \leq k \leq n(p-1)}\left\{\frac{(n p)^{\frac{2 k}{n}}}{2^{\frac{k}{n}}(k !)^{\frac{1}{n}}}\left[C_{2}+\int_{0}^{\infty} e^{-z} z^{n p-k-n} d z\right]\right\} \\
& \leq C_{3} \max _{0 \leq k \leq n(p-1)} \frac{n^{\frac{2 k}{n}}}{(k !)^{\frac{1}{n}}}[(n p-k-n) !]^{\frac{1}{n}} \\
& \leq C_{4} \max _{0 \leq k \leq n(p-1)} \frac{n^{\frac{2 k}{n}} n^{\frac{n p-k-n}{n}}}{k^{\frac{k}{n}}}=C_{4} \max _{0 \leq k \leq n(p-1)}\left(\frac{n}{k}\right)^{\frac{k}{n}} n^{p-1} .
\end{aligned}
$$

Since the function $x^{\frac{1}{x}}$ is bounded for $x>0,\left(\frac{n}{k}\right)^{\frac{k}{n}} \leq C_{5}, 0 \leq k \leq n p-n$ and $T^{\frac{1}{n}} \leq C_{6} n^{p-1}$. The lemma is proved.

Proof of Theorem 1. Let

$$
\begin{aligned}
E_{1}= & \left\{\int_{0}^{\infty} e^{-a t^{p}} x\left(t, x_{0}\right) d t \mid x\left(t, x_{0}\right)\right. \\
& \text { is a solution to (2), } \left.\quad a>\alpha\left(x_{0}\right), x_{0} \in E\right\},
\end{aligned}
$$




$$
x_{\varepsilon}=c_{\varepsilon} \int_{0}^{\infty} e^{-t^{p} / \varepsilon} x\left(t, x_{0}\right) d t, \quad c_{\varepsilon}=p \varepsilon^{-1 / p} \Gamma\left(\frac{1}{p}\right)^{-1}, \quad \frac{1}{\varepsilon}>\alpha\left(x_{0}\right) .
$$

The following estimates demonstrate that the elements of $E$ can be approximated by the elements of $E_{1}$.

For arbitrary $x_{0} \in E$,

$$
\begin{gathered}
\left\|x_{\varepsilon}-x_{0}\right\| \leq c_{\varepsilon} \int_{0}^{\infty} e^{-t^{p} / \varepsilon}\left\|x\left(t, x_{0}\right)-x_{0}\right\| d t \\
\leq \sup _{0 \leq t \leq \varepsilon}\left\|x\left(t, x_{0}\right)-x_{0}\right\|+c_{\varepsilon} \int_{t>\varepsilon}^{\infty} e^{-t^{p} / \varepsilon}\left(\left\|x_{0}\right\|+e^{\alpha t^{p}}\right) d t .
\end{gathered}
$$

Using the continuity of $x\left(t, x_{0}\right)$ at 0 , we have $x_{\varepsilon} \rightarrow x_{0}, \varepsilon \rightarrow 0$. The last statement and condition $(G)$ imply that $E_{1}$ is dense in $X$. Further, condition $(G)$ and integration by parts yield for $y \in E_{1}$ that $y \in D(A)$ and

$$
A y=(-1)^{p} \int_{0}^{\infty}\left(e^{-a t^{p}}\right)^{(p)} x\left(t, x_{0}\right) d t
$$

By induction,

$$
A^{n} y=(-1)^{n p} \int_{0}^{\infty}\left(e^{-a t^{p}}\right)^{(n p)} x\left(t, x_{0}\right) d t
$$

By Lemma 1,

$$
\left\|A^{n} y\right\|^{\frac{1}{n p}}=o(n), \quad n \rightarrow \infty .
$$

Thus the function $\hat{x}(t, y)=\sum_{n=0}^{\infty} \frac{t^{p n} A^{n} y}{(p n) !}$ is entire. Moreover, $\hat{x}(t, y)$ is a solution of the problem (2).

Remark 4. It follows from (16) that the function $\tilde{x}(t, y)=\sum_{n=0}^{\infty} \frac{t^{p n} A^{n} y}{(n !)^{p}}$ is also entire for $y \in E_{1}$. Observe that $\tilde{x}\left(\left(t_{1} \ldots t_{p}\right)^{1 / p}, y\right)$ is the strong solution of the Goursat problem

$$
\frac{\partial^{p} x}{\partial t_{1} \ldots \partial t_{p}}=A x, x(\overrightarrow{0})=y, \quad p \geq 2 .
$$

So the following corollary is true.

Corollary 1. Under the condition $(G)$ on the operator $A$ there is a dense subset $E_{1}$ in $X$ such that for every $y \in E_{1}$ the Goursat problem (17) has a strong solution.

(Concerning the existence of strong solutions to abstract Goursat problems, see $[7,4,8])$.

The next corollary modifies the statement of Theorem 1 for Cauchy problems of the form (1). 
Corollary 2. Let the operator $A$ satisfy the condition $(G), p \geq 2$. Then the set

$$
\begin{aligned}
& \left\{\vec{x}_{0}=\left(x_{i}\right)_{i=0}^{p-1} \mid \text { the solution } x\left(t, \vec{x}_{0}\right) \text { of }(1)\right. \text { exists and can be } \\
& \text { extended to an entire function } x(z): \mathbb{C} \rightarrow X\}
\end{aligned}
$$

is dense in $\bigoplus_{i=1}^{p} X$.

The result follows immediately from the representation

$$
x\left(t, \vec{x}_{0}\right)=\sum_{i=0}^{p-1} \sum_{n=0}^{\infty} \frac{t^{n p+k}}{(p n+k) !} A^{n} x_{i}
$$

$x_{i} \in E, 0 \leq i \leq p-1$.

\section{ACKNOWLEDGMENT}

The authors would like to thank the referees for their helpful suggestions and remarks.

\section{REFERENCES}

[1] R. Beals, Semigroups and abstract Gevrey spaces, J. Funct. Anal. 10, (1972), 300-308. MR 50:14355

[2] I. Cioranescu, U. Neumann, Stielties vectors of cosine functions generators, Studia Math.87, (1987), 1-7. MR 89d:47087

[3] H. O. Fattorini, Ordinary differential equations in linear topological spaces I, J. of Diff. Eq. 5, (1969), 72-105. MR 43:3593

[4] H. O. Fattorini, The abstract Goursat problem, Pacific. J. Math. 37, (1971), 51-83. MR 46:9793

[5] Yu. I. Lyubich, The classical and local Laplace transform in the abstract Cauchy problem, Russian Math. Surveys 21, (1966), no. 3, 1-52. MR 33:7889

[6] S. Nelson, R. Triggiani, Analytic properties of cosine operators, Proc. Amer. Math. Soc. 74, (1979), 101-104. MR 80h:47051

[7] J. Spellmann, Concerning the infinite differentiability of semigroup motions, Pacific. J. Math. 30 (1969), 519-523. MR 40:3360

[8] W. Roth, Goursat problem for $u_{r s}=$ Lu, Indiana Univ. Math. J. 22, (1973), 779-788. MR 46:9568

Department of Mathematics, Kiev University, 64 Vladimirskaya str., Kiev 252601, UKRAINE

E-mail address: mishura@math.carrier.kiev.ua

E-mail address: tomilov@math.carrier.kiev.ua 